\title{
Linx
}

Revue des linguistes de l'université Paris X Nanterre

70-71| 2014

Variations sémantiques et syntaxiques : aspects d'une théorie de l'invariance

\section{Le passif anglais BE+EN et l'objet affecté}

\section{Gérard Mélis}

\section{(2) OpenEdition}

\section{Journals}

Édition électronique

URL : http://journals.openedition.org/linx/1578

DOI : $10.4000 /$ linx. 1578

ISSN : 2118-9692

Éditeur

Presses universitaires de Paris Nanterre

\section{Édition imprimée}

Date de publication : 1 septembre 2014

Pagination : 185-196

ISSN : 0246-8743

\section{Référence électronique}

Gérard Mélis, «Le passif anglais BE+EN et l'objet affecté », Linx [En ligne], 70-71 | 2014, mis en ligne le 01 mars 2015, consulté le 05 mai 2019. URL : http://journals.openedition.org/linx/1578 ; DOI :

10.4000/linx.1578 


\section{Le passif anglais $\mathrm{BE}+\mathrm{EN}$ et l'objet affecté}

Gérard Mélis, Université Paris Diderot

Sorbonne Paris Cité, CLILLAC-ARP

\section{Introduction}

Bien que le passif anglais en $\mathrm{BE}+\mathrm{EN}$ soit prototypiquement associé à la notion d'«objet affecté », il existe un grand nombre de cas où cette notion ne semble pas s'appliquer : le contenu placé en position de sujet $S$ dans une phrase passive ne renvoie pas systématiquement à une entité modifiée dans sa matérialité. C'est à partir de ce

constat que nous posons la problématique de «l'objet affecté»( $(\mathbb{1})$. Les formes passives que nous examinons ne renvoient pas toutes à la modification matérielle d'un objet : dans ce cas, pourquoi le passif est-il tout de même applicable ? Nous abordons cette question sous deux angles.

D’une part, il nous semble nécessaire d'établir qu'une relation sujet-objet se construit à travers un verbe afin de rendre le passif possible. Ceci nous conduit à nous interroger sur le passif prépositionnel anglais, qui, à première vue, permet la passivation non à partir d'un complément mais d'un adjoint ( $\$ 2$ «Adjoint ou complément?»). Nous associons cette problématique syntaxique avec des considérations sémantiques exprimées en termes de relation serrée/relation lâche entre les notions présentes dans une relation : il apparaitra que la passivation repose sur une certaine congruence sémantique entre les contenus dans une relation, congruence qui agit comme une contrainte sur le passif. 
D'autre part, une fois établi que la passivation repose sur une relation sujetobjet, même dans les cas où cette relation semble ne pas apparaître, nous abordons la question de la définition des niveaux auxquels l'altération de l'objet peut intervenir ( $\$ 3$ «Les plans d'altération de l'objet»). Dans ce cadre, le contenu thématisé au passif est toujours le support d'une modification, mais elle se situe sur des plans différents selon les cas : elle peut concerner la représentation de l'objet et non sa matérialité, ou la situation dans laquelle il se trouve.

\section{La problématique de «l'objet affecté »}

Le passif en BE+EN est prototypiquement associé au fait que la fonction de sujet $\mathrm{S}$ de la proposition est remplie par un contenu qui fait référence à l'objet d'une transformation matérielle. Le passif prototypique s'illustre classiquement avec des exemples tels que :

(1a) Oswald assassinated Kennedy.

(1b) Kennedy was assassinated by Oswald. (Huddleston \& Pullum 2002, p. 1427)

(1c) Kennedy was assassinated in 1963.

Ces exemples se construisent sur une relation agent-patient: la voix permet d'attribuer aux termes occupant la position syntaxique de sujet $\mathrm{S}$ (argument externe) un de ces rôles d'argument: dans (1a), "[Oswald] is aligned with an active role, with the role of an agent : Oswald performed the action », et, dans (1b), «[Kennedy] has a passive role : Kennedy was the one on whom the action was performed » (Huddleston \& Pullum 2002, p. 1427).

La forme passive est liée aux notions de résultat et de modification. Le participe -EN a une valeur résultative (il est aussi employé dans le perfect anglais HAVE+EN) et la forme $\mathrm{BE}+\mathrm{EN}$ permet d'orienter l'énoncé sur l'état résultant d'un processus : « this participle is also used to describe the result of the activity (if it does have a definite result). A passive construction may be used in order to focus on this result, e.g. My neighbour was appointed to the board (by the managing director), The goalkeeper is being rested from next week's game, That cup was chipped by Mary when she washed the dishes.» (Dixon 2005, p. 367)

Les énoncés actifs et les énoncés en BE+EN constituent des modes distincts de représentation. L'énoncé peut faire référence à un état de fait ou bien présenter la qualification d'un objet : avec le passif, l'objet est représenté dans un certain état ou une certaine situation. Ces deux modes ne subissent pas les mêmes contraintes, ce qui justifie des remarques du type: "Neither passivization nor subject-raising is a mechanical syntactic operation deriving one sentence type from another ; rather they represent separate constructions imposing their imagery and perspective on a scene. » (Langacker 1982, p. 75)

Avec le passif, on quitte le domaine de l'énoncé opératif (renvoi à un état de fait) au bénéfice du résultatif (attribution de propriété dans un énoncé de qualification) (Joly \& O'Kelly 1990, p. 150-168) : d'une part, la voix opérative renvoie à la production d'une occurrence de procès, et, d'autre part, la voix résultative indique que l'énoncé définit un état résultant provenant d'une occurrence. Dans le registre opératif, l'énoncé réfère à « l'événement rapporté au sujet de causation » (Joly \& O'Kelly 1990, p. 155). 
Dans le registre résultatif, la construction est un énoncé de qualification qui fait référence non à un état de fait mais à une propriété. La forme dite passive fait référence à un événement vu sous l'angle de la propriété qu'il génère. La différence entre "passif d'action » (version "passive » d'un énoncé "actif») et "passif d'état» (fonction adjectivale du participe -EN) tient à la possibilité de reconstruire ou non un événement produisant l'état résultant (événement noté ' $E$ ' dans cet article). Dans les deux cas, la forme $\mathrm{BE}+\mathrm{EN}$ indique une propriété du contenu placé en position de sujet S. L'opposition opératif/résultatif, de nature énonciative (définition de deux registres d'énoncé), permet de se dégager de l'opposition actif/passif, de nature référentielle, qui se base sur la relation agent-patient. Comme nous le verrons, cette relation prototypique ne se retrouve pas dans tous les cas de passivation.

D'autre part, la passivation a un sens en fonction d'une échelle hiérarchique d'accessibilité à la position sujet (Khalifa 2004, p. 36) qui se construit à partir d'un ensemble complexe de critères, notamment la nature référentielle des contenus en termes de caractère animé et/ou défini, et le statut thématique des contenus d'une relation tel qu'il est marqué par une position syntaxique particulière comme le sujet ou le complément (Comrie 1981, Croft 1990, Givón 1984, 1993, Kuno 1976, Palmer 1994). Cette échelle permet de prévoir quel argument d'une relation sera vraisemblablement placé en première position dans une phrase active. Dès lors que l'argument identifié comme premier argument d'une relation en fonction de ce type d'échelle n'occupe pas la position de sujet $\mathrm{S}$, le passif est utilisé afin de promouvoir un autre argument et le placer en S. Ce premier argument peut ne pas être mentionné dans un passif court sans complément en BY (1c) ou bien être intégré dans la structure dans un passif long en BY (1b). Nous employons ici la terminologie descriptive communément admise dans les grammaires, telles que Huddleston \& Pullum (2002).

De plus, la passivation modifie le statut discursif du contenu placé en $S$ : dans (1a) et (1b), le même contenu [Kennedy] a toujours le même rôle de patient, que la phrase soit active ou passive, mais le fait de le placer en position $\mathrm{S}$ oriente différemment l'énoncé. Le contenu placé en position S reçoit le statut informationnel particulier de contenu thématisé, et le passif permet de mettre en place une orientation thématique sur l'argument de la relation qui aurait été placé en seconde position à l'actif (Albrespit 1994, Birner \& Ward 1998, Dixon 2005, Huddleston \& Pullum 2002, Puckika 2003) : "Voice is to be understood as a mechanism that selects a grammatically prominent syntactic constituent - subject - from the underlying semantic functions (case or thematic roles) of a clause. » (Shibatani 1985, p. 824). On retrouve le même type d'idée chez Dixon (2005, p. 359).

Deux paramètres fondamentaux sont donc à l'œuvre dans la passivation : le rôle argumental du contenu en question dans le cadre d'une relation (référence à une entité modifiée), et le fait que la place de sujet $\mathrm{S}$ tend à être une position distinguée à l'intérieur de la proposition. Ces deux paramètres signifient que la modification de l'entité référentielle en question doit aussi être pertinente sur le plan discursif. Le fait que l'objet soit modifié n'est pas en soi suffisant, car l'objet est modifié même dans l'actif. En revanche, si l'objet n'est pas affecté, le terme qui le représente dans la phrase ne peut pas être thématisé avec le passif. Il est a priori nécessaire de réunir les deux conditions pour que le passif soit acceptable, ce qui nous conduit à la notion d'effet pertinent. 
Dans les cas tels que (1), il est facile d'identifier les rôles (agent / patient) et de voir qu'il y a effectivement changement d'état du patient. L'énoncé avec BE+EN exprime explicitement ce changement d'état. Cette relation peut se paraphraser sous la forme : «l'agent a un effet sur le patient par l'intermédiaire du procès 'assassinate' ». Cependant, toutes les formes passives BE+EN n'entrent pas dans ce cadre. Les exemples qui suivent ne correspondent pas à ce cas prototypique. En effet, la relation en (2) n'est a priori pas transitive, et l'idée de modification d'un objet n'est pas systématiquement pertinente avec des verbes comme sleep (2-5) ou visit (6) :

(2) This bed has been slept in.

(3) The cornflower-blue dress she had brought to wear at the finals had been slept on by Ethel and was impossibly creased, as was her face after two nights sleeping in the car.

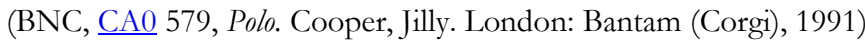

(4) Whare - Maori word for house. Most whares were small with a tiny door. As they were only considered as sleeping houses, they were rarely tall enough for a person to stand upright. They consisted of wooden frames and the walls and roof were of tightly woven dry grass and flax. The fireplace, in the middle enclosed by stones, was slept around. Most whares had a verandah.

(Albrespit, 2007, p. 473)

(5) The bed was slept in by George Washington.

(Huddleston 2002, p. 1446)

(6) The workhouse will be visited by one or more very important people; perhaps a magistrate, an inspector of workhouses, or a wealthy benefactor.

(BNC AM6 692, Drama as education. Bolton, Gavin. Harlow: Longman Group UK Ltd, 1988)

Il est donc légitime de se demander comment le passif est tout de même applicable à ces cas non prototypiques, et ce que devient la notion d'objet affecté dans ces différents cas. En effet, des structures telles que «The English Channel was first swum in 1875 », ou "French is spoken throughout my chain of boutiques » (Dixon 2005, p. 372) n'indiquent pas que le référent du sujet $\mathrm{S}$ a été affecté par une action.

\section{Adjoint ou complément?}

Le passif prépositionnel illustré dans (2) semble contredire l'idée que seul un complément du verbe peut se placer en $\mathrm{S}$ avec la forme passive : apparemment, un adjoint peut occuper la place $S$ dans un passif. Or, cette possibilité n'est pas systématiquement possible en anglais :

(7) John slept in the garden.

(8) *?The garden was slept in (by John).

(9) John worked yesterday.

(10)* Yesterday was worked (by John). 
Afin de justifier des constructions telles que (2), il est possible d'envisager plusieurs manières d'analyser la structure syntaxique de la construction et d'interpréter ce qui passe a priori pour un adjoint en tant que complément du verbe.

On peut s'interroger sur la relation entre les arguments dans une relation de type (11):

(11) John slept in this bed.

Le syntagme prépositionnel [in this bed] est-il un adjoint qui fait référence à une localisation? Est-ce un complément du verbe au sens où il réfère à ce qui est directement affecté par un événement ?

L'énoncé (11) peut référer à une relation de type $<<$ état de fait $><$ localisation $>>$, auquel cas le syntagme prépositionnel est un adjoint, mais ce n'est pas la seule analyse possible. D'une part, (11) peut être la réponse à la question « Where did John sleep? ». D'autre part, cet énoncé peut correspondre à un autre contexte :

(12) - Why is the bed rumpled?

- John slept in it last night.

Dans ce cas, l'énoncé (11) peut se paraphraser de telle sorte qu'apparaisse l'altération d'un objet: John a un effet sur le lit dans le cadre de la relation $<$ John + sleep in this bed $>$. Ceci explique pourquoi il est possible de produire (2) alors que (13-17) sont plus difficilement acceptables :

(13) ???This chair has been slept on.

(14) ???This chair has been sat on.

(15) ????* That bed has been committed suicide in.

(16) ????* That bed has been painted a watercolour sketch in.

(17) ????* That bed has been written a letter in.

Dans (13-14), il est difficile d'imaginer un effet portant sur l'objet « the chair». En effet, a priori, le fait de dormir ou simplement de s'asseoir sur une chaise ne modifie pas cet objet d'une quelconque manière. La présence d'un verbe associé à son complément rend la passivation encore plus difficilement acceptable (15-17). On observe que la place de complément du verbe est saturée (complément de « write » ou de «commit»), et ne correspond pas au SN [that bed]. Quand le terme qui occupe la place de sujet $\mathrm{S}$ dans la forme passive n'est a priori pas le complément du verbe, la passivation est moins probable :

(18) ????* That bed has been written in.

Ici, l'objet modifié par le procès « write » demeure son argument interne implicite qui ne s'identifie pas à [the bed], d'où la faible acceptabilité de (18).

La relation syntaxique formulée en termes de complément peut aussi s'exprimer en termes sémantiques de relation plus ou moins serrée entre les termes d'une relation. La relation «bed-sleep » est plus resserrée que la relation «bed-write $\mathrm{x}$ » : la relation $<\mathrm{X}$ sleep in a bed $>$ est plus attendue que $<\mathrm{X}$ write in a bed $>$ etc..., et il est plus facile de concevoir un effet pertinent sur le lit à partir de cette relation attendue qu'avec d'autres relations : la relation entre «lit défait, draps froissés » et «quelqu'un a dormi dans le lit» est plus immédiate que d'autres sortes d'enchaînement. Plus la relation est lâche, moins la passivation est facilement acceptable. Syntaxiquement, la 
relation V-objet est plus resserrée que la relation $\mathrm{V}$-adjoint. Les deux niveaux d'analyse (syntaxique/sémantique) sont donc corrélables.

Cette relation serrée se manifeste en tant que relation qui met en évidence une congruence sémantique entre les termes impliqués dans la relation passivable. On retrouve ici le principe de présupposition cognitive mentionné chez De Giorgi (1997, p. 142) à propos d'exemples tels que (19) qui montrent que la congruence entre le procès et l'argument placé en position $\mathrm{S}$ est un paramètre dans la passivation :

(19) Of the three bedrooms I had inspected, one had obviously been slept in.

Ces phénomènes de congruence sémantique pourraient rendre compte de certaines impossibilités telles que (20):

(20) *The tree had been died near. (Huddleston \& Pullum 2002, p. 1434)

Ces énoncés se distinguent des relations «sleep-bed» dans (2) dans la mesure où la relation «die-tree» est moins facilement disponible, car il n'y a pas, dans ce dernier cas, de présupposition cognitive. Le paramètre de la modification matérielle de l'objet converge avec la question des propriétés primitives : en (20), il n'y a pas d'effet sur l'arbre dans sa matérialité, et la relation « die-tree » n'est pas de l'ordre de l'attendu. De même, si l'énoncé «*The river was slept beside. » n'est pas acceptable, alors que (19) est possible, c'est parce que « sleeping beside a river doesn't affect it or distinguish it in any significant way from other rivers. » (Huddleston \& Pullum 2002, p. 1147) : la relation «sleep-river », qui n'est pas un cas de présupposition cognitive, ne permet pas non plus d'établir de lien causal entre l'événement « Someone slept beside the river » et une modification quelconque du cours d'eau.

Il est cependant possible d'orienter un énoncé vers l'idée de résultat alors que la relation entre le procès et l'argument en position $\mathrm{S}$ n'est pas serrée :

Examples are found where the preposition does have its locative sense: « The bed had been slept in », "Her hat had been sat on »; for the passive to be acceptable in such cases, the process must be one that affects in some significant way the referent of the subject. Thus "Her hat had been sat on » is more likely than « The stone bench has been sat on ", for sitting on a hat is likely to put it out of shape, whereas sitting on a stone bench will not normally have any effect on it - though (...) if one adds plainly, acceptability is improved: the plainly indicates that there was some visible effect produced by sitting on the bench.

(Huddleston 1984, p. 441)

Néanmoins, ce type de manipulation n'améliore pas vraiment la grammaticalité des exemples du type (20):

(21) ???* This tree has plainly been died near.

(22) ???* This tree has plainly been died under.

L'état résultant exprimé par le passif est évident dans « Her hat had been sat on» ou en (3) («The cornflower-blue dress (...) had been slept on by Ethel and was impossibly creased») : l'état de l'objet («creased») est clairement provoqué par l'événement < Ethel slept on the dress $>$. Un adverbe tel «plainly » peut, dans certains cas, mettre en avant cette modification d'état. Néanmoins, il faut tout de même que la 
relation procès-argument soit sémantiquement compatible avec la production d'un effet, ce qui justifie la faible acceptabilité de (21) et (22).

Le passif prépositionnel semble donc contraint par trois paramètres : a / la relation entre le procès et l'argument placé en position $S$ doit être de l'ordre de l'attendu (relation primitive, présupposition cognitive), b/ le S.Prep est interprétable comme complément du verbe, ce qui signifie ici qu'il y a un effet sur cet argument, c / cet effet doit être pertinent dans un contexte discursif donné.

Il reste à définir, dans le cadre de cette relation sujet-objet, les différents types d'effet portant sur l'objet: dans la section suivante, nous avancerons l'idée que le passif thématise un contenu affecté et que les différences d'interprétation tiennent au fait que l'objet peut être affecté sur différents plans.

\section{Les plans d'altération de l'objet}

Notre hypothèse est qu'il existe différentes réalisations de la relation « avoir un effet sur ». En plus du cas où un objet est matériellement altéré par l'action d'un agent (1), il est possible de définir au moins deux autres cas : a / l'objet est altéré dans sa représentation, b / la situation dans laquelle se trouve l'objet est altérée.

\subsection{Représentation d'objet}

Nous défendons ici l'hypothèse selon laquelle le passif indique un changement d'état du contenu thématisé en position $S$, même quand cette modification n'est pas matérielle : dans ce cas, c'est la représentation de l'objet, et non l'objet lui-même dans sa matérialité, qui est concernée.

Dans un cas comme (5) («The bed was slept in by George Washington.»), l'absence d'effet matériel sur l'objet ne bloque pas la passivation. On retrouve l'idée de modification d'objet placée cette fois-ci sur le plan de la conceptualisation de cet objet. L'état de fait $\mathrm{E}$ « $\mathrm{GW}$ slept in this bed. » rend remarquable l'objet impliqué dans cette relation: c'est un lit historique, ce n'est pas n'importe quel lit, car il a été mis en contact avec une personnalité marquante. L'objet change de statut conceptuel du fait de l'événement E, ce qui justifie le passif. Il est ici nécessaire de préciser l'identité du premier argument de la relation, car c'est justement le statut de cet argument qui permet à l'autre argument d'être modifié dans la manière qu'il a d'être conceptualisé : il est distingué qualitativement en fonction de la relation $\mathrm{E}$.

La différence entre modification matérielle et modification conceptuelle permet de poser la question du complément en BY (passif long). Dans les exemples du type (2) ("This bed has been slept in.»), il n'est pas nécessaire de mentionner le premier argument du procès : l'identité particulière ne change rien à l'état matériel du lit (que ce soit John ou quelqu'un d'autre, le résultat est le même : les draps sont froissés, le lit est défait), d'où la tendance à utiliser le passif court dans ce type de structure, même si le passif long est possible, comme dans (3) («The dress had been slept on by Ethel and was impossibly creased.»). En revanche, l'identité du meurtrier en (1) (« Oswald assassinated Kennedy. ») est significative, ce qui permet le passif long. Dans (5), c'est la personnalité historique qui permet de distinguer le lit, d'où le besoin de produire un passif long. 
En cas de passif long, le SPrép peut contenir une simple information, comme dans (3). Mais le SPrép peut aussi contenir une information remarquable, comme dans (5) (référence à une personne connue), auquel cas il est nécessaire de changer de niveau de représentation. Pourquoi en effet dire que c'est G. Washington en personne qui a dormi dans le lit pour simplement dire que le lit est défait alors que n'importe quelle autre personne peut produire le même résultat ? Si une personne remarquable est représentée dans l'énoncé, c'est pour assigner à l'objet un statut conceptuel particulier.

Dans des énoncés tels que (4) ("The fireplace, in the middle enclosed by stones, was slept around»), l'énoncé au passif ne fait pas référence à une occurrence particulière : il définit une propriété constitutive du «fireplace ». L'énoncé exprime une manière de concevoir l'objet: il s'agit d'un dispositif qui est configuré de telle sorte que les habitants dorment installés autour de lui. L'état de fait représenté par la relation $\mathrm{E}<$ people + sleep around the fireplace $>$ ne modifie pas l'objet dans sa matérialité mais il en fait un objet doté de certaines fonctions, de certaines caractéristiques, d'une certaine identité. Il est conçu (matériellement et conceptuellement) d'une certaine manière et se distingue qualitativement grâce à la relation $\mathrm{E}$.

D'autre part, le dispositif discursif installe le bâtiment (et ses différentes composantes tels que les murs, le toit, le foyer, la véranda) en tant qu'objet de discours, au détriment du premier argument du verbe « sleep » (les « sleepers »), ce qui explique l'emploi d'un passif court. Dès le début de la séquence, il est établi que le thème de discours est la notion de "whare » : tous les éléments qui en font partie sont thématiques et ils servent de support à des apports prédicatifs qui constituent des nouvelles informations fournies au destinataire (la taille des "whares », leur architecture, la matière dont sont faits les murs et le toit, la position et la fonction du "fireplace ») : cette progression thématique modifie la représentation du contenu représenté par le sujet $\mathrm{S}$ du passif car il y a, dans ces énoncés, un apport informationnel.

\subsection{Objet et situation d'objet}

Dans (6) («The workhouse will be visited by one or more very important people »), le bâtiment n'est pas modifié matériellement par l'état de fait $\mathrm{E}<$ one or more very important people + visit the workhouse $>$ et il ne s'agit pas d'en donner une représentation particulière comme cela pourrait être le cas dans :

(23) The workhouse has been visited by the Emperor himself.

Cet énoncé (23) pourrait s'approcher de (5) car il signifie que l'on parle d'un bâtiment particularisé, l'Empereur ne se déplaçant pas n'importe où.

L'exemple (6) présente un cas d'altération non de l'objet lui-même mais de la situation dans laquelle il peut se trouver. L'établissement est dans son état normal s'il n'y a pas de visite ou d'inspection, mais la visite le place dans des circonstances particulières au sens où quelque chose le concernant peut en résulter, avec des effets possibles en fonction de l'identité du visiteur : un inspecteur peut évaluer positivement ou négativement l'efficacité du lieu mais un bienfaiteur pourra faire un don, ... etc, d'où le passif long. L'identité du premier argument est pertinente dans la mesure où 
elle permet de distinguer les situations dans lesquelles l'objet peut se trouver. Un énoncé tel que (24) n'est envisageable que si le référent du SN [John] constitue un seuil de différenciation de ces situations :

(24) The workhouse will be visited by John.

En cas contraire, la forme active «John will visit the workhouse » sera préférée. Notons que la question de savoir précisément et formellement quelle notion peut servir de seuil de différenciation reste ouverte. Néanmoins, il reste possible d'estimer intuitivement que tel ou tel contenu peut servir à distinguer qualitativement un état de fait $\mathrm{E}$, et rendre ainsi possible le passif.

Nous voyons que la forme passive opère une thématisation d'un terme renvoyant à un élément affecté, et que cette modification fonctionne à différents niveaux. Elle peut être matérielle et s'effectuer au niveau référentiel de l'énoncé (dans (2), (3) ou (1b), l'énoncé fait référence à un événement qui altère un objet). Il peut aussi s'agir d'une modification de conceptualisation de l'objet à travers un événement (5), ou bien d'une modification de conceptualisation de l'objet en tant que thème de discours dans une cadre textuel : dans (4), c'est l'apport prédicatif sur un support thématique qui constitue la modification conceptuelle du support. Nous retrouvons donc dans tous ces cas l'idée d'altération, mais à des niveaux différents. Elle peut agir à un niveau strictement référentiel dans (2) (un événement modifie l'état d'un objet), mais aussi à un niveau plus conceptuel. Dans (5), un événement modifie l'appréciation sur un objet, et, dans (4), c'est l'apport d'information sur le thème qui modifie la représentation de l'objet. La modification peut aussi porter sur la situation dans laquelle se trouve l'objet représenté par le sujet $\mathrm{S}$ : dans la phrase passive (6), cette modification permet l'ajout d'un apport nouveau en ce qui concerne le support prédicatif thématisé (étant donné l'état de fait $\mathrm{E}$, le référent de $\mathrm{S}$ est placé dans une nouvelle situation).

Ces hypothèses permettent de rendre compte de certains faits, illustrés dans les énoncés (25-28) :

(25) The English Channel was first swum in 1875.

(26) French is spoken throughout my chain of boutiques.

(27)* The English Channel was first swum across in 1875.

(28) * French is spoken in throughout my chain of boutiques.

(Dixon, 2005, p. 372)

Bien que le référent du $\mathrm{S}$ ne soit pas ici modifié dans sa matérialité, la passivation est non seulement possible mais elle produit des significations particulières qui ne sont pas comparables avec les interprétations de l'actif. L'énoncé actif «Matthew Webb swam in the English Channel in 1875 » ne fait que repérer une occurrence dans le temps, alors que (25) signifie que quelque chose de particulier s'est passé à propos du référent de $\mathrm{S}$. En effet, un exploit sportif a montré que la Manche est franchissable, ce qui n'était pas le cas avant : la représentation du référent de $S$ est modifiée.

Dans l'énoncé (25), la Manche est représentée comme une distance à parcourir à la nage, comme dans les exemples :

(29) It takes a few moments to adjust — one moment one is dry, wearing dry swimming trunks and walking about, and the next one is progressively getting wetter (including the swimming trunks, which somehow seems particularly inappropriate) 
— and it's only when half a length or so has been swum that the whole thing seems ordinary again.

(BNC EVA788, Sleep and dreaming. Empson, Jacob. London: Faber \& Faber Ltd, 1989)

(30) Each year during the 1920s and 1930s, a one-mile race between the bathhouse at English Bay and Kitsilano Beach was swum by open water enthusiasts.

(Google, Vancouver Open Water Swim Association, 16/6/2012)

Dans ce type de cas, on retrouve le principe de présupposition cognitive. En effet, la relation entre le contenu du S (la distance « half a length or so » en (29) et, en (30), la « one-mile race between... Beach ») et le procès «swim » est déjà construite : dans le contexte de (29), il s'agit de définir une longueur à parcourir à la nage, et, en (30), le contexte fait déjà référence à l'activité de nager (le site en question est consacré à cette activité), et le $\mathrm{S}$ réfère à une course, qui ne peut ici concerner que la natation. De plus, l'énoncé (30) fait référence non à un événement singulier mais à ce qui se passe régulièrement ("Each year during the 1920s and 1930s »), ce qui permet d'attribuer une propriété («be swum by open water enthusiasts») au $\mathrm{S}$ «a one milerace between... Beach» dans le cadre d'une compétition. Enfin, le fait de parcourir une longueur jusqu'au bout modifie la situation qui intègre cette distance. En effet, le contexte est explicite sur ce point: avant d'atteindre la fin de cette distance il y a encore des efforts d'adaptation à fournir («It takes a few moments to adjust»), mais une fois cette distance parcourue, ce n'est plus le cas ( $"$ the whole thing seems ordinary again »).

Il en va différemment dans (27). Cet énoncé avec «across » ne rend pas le passif acceptable ; il en irait de même avec « in » (* In 1875, The English Channel was first swum in »). Ces énoncés indiquent qu'une action particulière faite par un agent particulier a été accomplie («Matthew Webb swam across / in the English Channel in $1875 »)$ sans pour autant produire un effet particulier sur la Manche à quelque niveau que ce soit. Les S.Prep localisent l'événement ou l'agent. Avec «in», la fonction sémantique du S.Prep est de localiser l'occurrence ("Matthew Webb swam») dans l'espace (le S.Prep a ici un statut d'adjoint). Avec «across », le S.Prep localise l'entité «M. Webb» dans l'espace (« M. Webb reached the other side of the Channel »). Dans ces cas, le passif n'est pas acceptable : le S.Prep avec « in » a un statut d'adjoint et non de complément, et le S.Prep avec « across » indique un changement de localisation du premier argument sans que le contenu du syntagme adjoint en soit altéré.

L'opposition entre (26) et (28) est significative elle aussi. L'énoncé actif du type «Shop assistants speak French throughout my chain of boutiques » n'est pas comparable avec (26) : il renvoie à ce que font les vendeurs dans les magasins de cette chaîne, et il peut produire une interprétation absurde (les vendeurs de mes magasins pratiquent le français pendant leurs heures de travail dans mes boutiques). En revanche, dans un énoncé tel que (26), il s'agit de définir une propriété pertinente du S «French» dans un cadre donné, afin d'établir par inférence que les magasins en question sont particuliers en ceci qu'ils peuvent recevoir une clientèle francophone. Un énoncé comme (28) indique la manière de s'exprimer de certaines personnes («Shop assistants speak in French »), ce qui fait du premier argument de la relation 
organisée autour de "speak» un support de qualification, et cela empêche le passif avec «French » en tant que $S$.

On note d'autres phénomènes intéressants illustrés dans (31-34):

(31) They could march through the valley in less than 2 hours.

(32) The valley could be marched through in less than 2 hours.

(33) They could march in the valley for less than 2 hours.

(34) *The valley could be marched in for less than 2 hours.

(Huddleston, Pullum, 2002, p. 1446)

L'énoncé (32) est possible car «if the valley could be marched through in less than 2 hours, this tells us something significant about its length and the terrain » (Huddleston \& Pullum, 2002, p. 1446) : le S « the valley » est la cible d'un commentaire pertinent dans (31) mais pas dans (34).

Avec «march through », l'aboutissement du processus dépend soit de l'agent, ce qui est exprimée avec la forme active dans (31), soit d'un objet qui facilite ou empêche cet aboutissement, ce que signifie (32). Dans la forme passive (32), c'est en fonction des caractéristiques de cet objet (longueur de la vallée, nature du terrain) que l'événement peut avoir lieu: cet énoncé définit donc des propriétés de «the valley », ce qui rend possible le passif, et il permet de représenter le contenu «the valley » d'une certaine manière (en tant que ce qui permet l'état de fait $\mathrm{E}$ ), ce qui n'est pas le cas dans (34).

C’est la préposition qui produit une différence sémantique décisive. Dans (31), le complément de mesure temporelle est introduit par «in », ce qui marque une télicité (la préposition marque le temps qu'il faut pour atteindre l'objectif), et le processus est associé à un aboutissement exprimé par «through » (d'un bout à l'autre, une traversée). En revanche, dans (33), «march in the valley» correspond à la localisation spatiale d'un événement, dont la durée peut être calculée par le syntagme en «for» (et non en « in ») : il y a une simple localisation d'un état de fait, et le S.Prep « in the valley » a un statut d'adjoint. L'événement «they could march in the valley» n'induit aucun changement matériel ou conceptuel du référent de «the valley », ce qui bloque le passif.

\section{Conclusion}

La forme BE+EN correspond dans tous les cas étudiés dans cet article à la modification d'un référent dont la représentation linguistique sert d'objet de discours pertinent. Cette modification se situe à différents niveaux : l'énoncé peut référer à un état de fait événementiel (modification matérielle), ou bien à une conception mentale (modification conceptuelle). Cette modification peut concerner l'objet directement, sa représentation, ce qui en est dit, ou bien la situation dans laquelle il se trouve. 


\section{Bibliographie}

Aikhenvald, A.Y., 2004, Evidentiality, Oxford, Oxford University Press.

Albrespit, J., 1994, "Manifestations de la diathèse passive en anglais contemporain dans le cadre de la théorie des opérations énonciatives ", Thèse, Université Paris VII-Denis Diderot.

Albrespit, J., 2007, «Atypical Passives », Études anglaises, 4/2007, Paris, Klincksieck / Didier Érudition, p. 466-482.

Birner, B, J., WARD, G., 1998, Information Status and Noncanonical Word Order in English, Amsterdam / Philadelphie, John Benjamins.

Comrie, B., 1981, Language Universals and Linguistic Typology, Chicago, The University of Chicago Press.

Croft, W., 1990, Typology and Universals, Cambridge, Cambridge University Press.

DE GIORGI, B., 1997, «Le passif en anglais - présupposition, information et équilibre thématique » in Anglophonia 2/1997, Presses Universitaires du Mirail, p. 127-157.

Dixon, R.M.W., [1991] 2005, A Semantic Approach to English Grammar, Oxford, Oxford University Press.

GIVÓN, T., 1984, « Direct object and dative shifting: semantic and pragmatic case », in Plank, F., (éd.), Objects, Londres, Academic Press, p. 151-182.

GIVÓN, T., 1993, English Grammar. A Function-Based Introduction, Amsterdam, John Benjamins.

HudDlEston, R., 1984, Introduction to the Grammar of English, Cambridge, Cambridge Textbooks in Linguistics.

Huddleston R., Pullum G. K., 2002, The Cambridge Grammar of the English Language, Cambridge, Cambridge University Press.

Joly A., O’Kelly, D., 1990, Grammaire systématique de l'anglais, Paris, Nathan.

KHALIFA, J.-C., 2004, Syntaxe de l'anglais - théories et pratiques de l'énoncé complexe, Gap, Ophrys.

KUNO, S., 1976, «Subject, theme and the speaker's empathy : a reexamination of relativisation phenomena », in Charles L., (éd.) Subject and Topic, New York Academic Press, p. 417444.

LANGACKER, R. W., 1982, «Space Grammar, Analysability, and the English Passive », Language, 58-1, p. 22-80.

Palmer, F., 1994, Grammatical Roles and Relations, Cambridge: Cambridge Textbooks in Linguistics, Cambridge University Press.

PUCKIKA, J., 2003, «La Diathèse en anglais contemporain : formes, valeurs et sens. Essai de redéfinition d'une catégorie grammaticale », Thèse, Université Bordeaux 3.

Shibatani, M., 1985, « Passives and Related Constructions: A Prototype Analysis », Language, 61-4, Dec 1985, p. 821-848. 